Check for updates

Cite this: RSC Adv., 2017, 7, 38300

\title{
One-step fabrication of a tunable nanofibrous well insert via electrolyte-assisted electrospinning $\dagger$
}

\author{
Seongsu Eom, (D) t $^{\mathrm{a}}$ Sang Min Park,,$^{\mathrm{a}}$ Seon Jin Han, ${ }^{\mathrm{a}}$ Joon Wan Kim ${ }^{\mathrm{b}}$ \\ and Dong Sung Kim (iD *a
}

The integration of the Transwell $\circledast$ assay with an electrospun nanofiber membrane shows a significant potential in chemotactic assays and co-culture models, but the complicated integration processes often limit its utilization. Here, we present a one-step fabrication process of a nanofibrous well insert by adopting electrolyte-assisted electrospinning, named ELES. The utilization of ELES, which introduced the electrolyte solution as a temporal collector, enabled the facilitation of not only the fabrication of a freestanding electrospun polycaprolactone (PCL) nanofiber on the bottom opening of a well insert wall but also the spontaneous integration between the nanofiber membrane and the well insert wall in a onestep process. The versatility of this approach was demonstrated by modulating the diameter of $\mathrm{PCL}$ nanofibers and thickness of the membrane. The indentation test revealed stable integration between the membrane and the well insert wall. The fabricated nanofibrous well inserts were confirmed as an in vitro cell culture platform with promising cell culture results of mouse brain endothelial cell line (bEnd.3).

Received 14th June 2017

Accepted 30th July 2017

DOI: $10.1039 / \mathrm{c} 7 \mathrm{ra06629e}$

rsc.li/rsc-advances

culture platform containing a nanofibrous membrane that

\section{Introduction}

A Transwell $®$ insert or modified Boyden chamber, which is composed of two sides separated by a permeable membrane, is the most widely accepted in vitro cell culture platform to observe and analyze cell migration and a co-culture model. Given that the dynamic interaction among multicellular components (e.g., cell migration) plays a key role in many biological phenomena, a well insert has significant opportunities to broaden the understanding of pathological phenomena including cancer metastasis, ${ }^{1,2}$ immune response, ${ }^{3}$ atherosclerosis, ${ }^{4,5}$ wound healing, ${ }^{6,7}$ and nutrient and protein transports. ${ }^{8}$ Though the commercially available Transwell ${ }^{\circledR}$ inserts have considerable accessibility, ease of control, and low cost, a 2D culture model on the flat porous surface provides limited understanding on cell functions due to its distinct structural difference from in vivo cellular environments. While the microenvironment of human tissue possesses 3D nanofibrous structures of the extracellular matrix (ECM) that mainly consists of collagen nanofibrils, the conventional flat membranes with the randomly distributed nano-/micro-scale circular pores provided 2D culture models. In this regard, developing a Transwell

${ }^{a}$ Department of Mechanical Engineering, Pohang University of Science and Technology (POSTECH), 77 Cheongam-Ro Nam-Gu, Pohang, Gyeongbuk, 37673 South Korea. E-mail: smkds@postech.ac.kr

${ }^{b}$ Institute of Innovative Research (IIR), Tokyo Institute of Technology (TOKYO TECH), J3-12, 4259 Nagatsuta-cho, Midori-ku, Yokohama, 226-8503 Japan

$\dagger$ Electronic supplementary information (ESI) available. See DOI: $10.1039 / \mathrm{c} 7 \mathrm{ra06629e}$

$\$$ These authors contributed equally to this work. mimics ECM structures is significant.

Electrospinning has been a widely utilized method to obtain ECM mimetic nanofibrous structures due to its simple system and versatile production of nanofibrous structures. Therefore, considerable effort to apply an electrospun nanofibrous structure on well insert to provide a more physiologically relevant environment has recently emerged. ${ }^{9-11}$ To apply electrospun nanofibers on a Transwell culture platform, the nanofibers should be interconnected into a 2D-nanofiber membrane and possess a free-standing structure. While the conventional electrospinning process is able to generate a non-woven $2 \mathrm{D}$ nanofiber membrane on the surface of a grounded metal collector, the membrane strongly adheres to the metal surface and thus lacks the free-standing structure. To achieve a free-standing nanofiber membrane, post-processes, such as mechanical peeling off, transfer, and attachment to a support, are required. Warnke et al. developed a retinal pigment epithelium model by utilizing a free-standing, circular-shaped nanofiber membrane, which, however, required peeling off an electrospun nanofiber membrane from the metal surface and cutting, followed by integration with ring-type support. ${ }^{9}$ Bischel et al. succeeded in the development of a bilayer model of the blood-brain barrier on a free-standing nanofiber membrane integrated with a well insert wall. However, it also necessitated peeling off and cutting of the nanofiber membrane from a metal collector, followed by attaching the membrane on the well insert wall using a heated soldering iron. ${ }^{10}$ The need for direct fabrication of a freestanding nanofiber membrane and its integration to a well insert without any post-processes has been emphasized because 
peeling off, cutting, and attaching of a nanofiber membrane without damage or misalignment commonly requires delicate handling and skilled techniques. Slater et al. employed a nickel mesh with micro-sized rectangular pores to directly fabricate a free-standing nanofiber membrane on nickel mesh and integrated the nickel mesh on a well insert. However, the process is not cost-effective due to the use of expendable nickel mesh, and the micro-porous structure of the nickel mesh is undesirable during cell culture because it might influence the cell behaviour. ${ }^{11}$ In short, the major limitation for the previous studies is the complicated fabrication process that causes poor accessibility and cost effectiveness. As a solution, our group has recently developed a novel electrospinning process, named electrolyte-assisted electrospinning (ELES), which enables the facile fabrication of a free-standing nanofiber membrane by using an electrolyte solution as a temporal collector. ${ }^{12}$

Here, we developed a one-step fabrication process of a nanofiber membrane-integrated well insert, named a nanofibrous well insert, without any post-processes, such as peeling off, cutting, transferring, and attaching, by adopting the ELES process. The utilization of ELES enabled the generation of a free-standing nanofiber membrane on a well insert wall with spontaneous integration. Moreover, this approach facilitated the modulation of the nanofiber membrane properties of the nanofibrous well insert. We confirmed the ability to modify the nanofiber diameter, membrane thickness, membrane pore size, and mechanical property by adjusting the electrospinning time and the concentration of the polymer solution. The mechanical indentation tests not only characterized mechanical properties of a membrane but also verified the strong adhesion of nanofiber membrane with well inserts. The potential biological relevance of the nanofibrous well inserts was verified by comparing the results of live/dead staining images and transendothelial electrical resistance (TEER) values of mouse brain endothelial cell line (bEnd.3) layers cultured on both commercial Transwell® inserts and nanofibrous well inserts.

\section{Materials and methods}

\subsection{Fabrication of nanofibrous well inserts based on electrolyte-assisted electrospinning}

Polycaprolactone (PCL, $M_{\mathrm{n}}=80000 \mathrm{~g} \mathrm{~mol}^{-1}$ ), chloroform, and methanol were purchased from Sigma-Aldrich and used as received. Polydimethylsiloxane (PDMS) and curing agent (Sylgard 184) were obtained from Dow Corning. Transwell ${ }^{\circledR}$ inserts (12-well dish type, $0.4 \mu \mathrm{m}$ pore size, 3460) with polyester (PET) membranes were purchased from Corning Inc. A PCL solution was prepared by dissolving PCL pellets in a mixture of methanol and chloroform (1/1 vol/vol). Chloroform was selected for its good solubility of PCL. The low dielectric constant of chloroform $(\varepsilon=4.75)$ was compensated by choosing methanol as another solvent with taken into account its higher dielectric constant $(\varepsilon=33.64)$, given that the high dielectric constant of the polymer solution should be required to decrease the diameter of the electrospun fibers down to the nanoscale. ${ }^{13}$

Instead of using a metal electrode for the grounded collector, a $0.5 \mathrm{M}$ potassium chloride $(\mathrm{KCl})$ solution was used as the grounded collector for electrospinning. After removing the PET membrane, the well insert wall was placed upside down and a PDMS cylindrical block was used to seal the top opening of the well insert wall $\left(1.12 \mathrm{~cm}^{2}\right.$ insert membrane area, 12-well dish type). The $\mathrm{KCl}$ solution was poured in the well insert wall and connected to the ground. The well insert wall with the $\mathrm{KCl}$ solution was placed $25 \mathrm{~cm}$ below the metal needle. The PCL solution was loaded into a plastic syringe and dispensed through a 23-gauge metal needle. We have carefully chosen the diameter of the metal needle not only enabling the fabrication of nanoscale electrospun fibers but also avoiding the clogging problem at the needle tip, which might occur when the metal needle diameter becomes too small. ${ }^{14}$ A syringe pump (KDS200, KD Scientific Inc.) fed the solution at a constant flow rate $\left(0.5 \mathrm{~mL} \mathrm{~h}^{-1}\right)$. Then, ELES was performed in an environmentcontrolled glove box where a high-voltage power supply (HV30, NanoNC) was utilized to provide a high voltage (19 kV) between the needle and the $\mathrm{KCl}$ solution collector. Once the electrospun PCL nanofiber membrane on the bottom opening of well insert wall was fabricated, the PDMS cylindrical block and the electrolyte solution were removed.

\subsection{Nanofiber mat characterization}

Scanning electron microscopy (SEM) (SU6600, Hitachi) was used to examine fiber diameters of electrospun nanofiber membrane. The diameter of nanofibers and the pore size of the nanofiber membrane were measured by using Image J (National Institutes of Health, USA) based on the SEM image. The pores were randomly chosen in the SEM image and the mean pore diameter $(D)$ was determined by using $D=[(4 \times A) / \pi]^{1 / 2}$, assuming circular pores, where $A$ is the average pore area. ${ }^{15}$ Though it was difficult to identify the exact shape and size of the pores of a nanofiber membrane because the pores are consequences of the interconnection of multi-stacked nanofibers, the assumption of the circular shape of the pore would provide not a precise, but a practical way to characterize the pore size of the nanofiber membrane. To measure the thickness of the nanofiber membrane, a mixture of PDMS with the curing agent in a $10: 1$ weight ratio, which was thoroughly stirred and degassed in a vacuum chamber, was poured to the nanofiber membrane covering the whole membrane, and then baked for $24 \mathrm{~h}$ on a $50{ }^{\circ} \mathrm{C}$ hot plate. The nanofiber membrane fixed by the cured PDMS was cross-cut and its cross-sectional surface was examined by an optical microscope. Then, based on the crosssectional image of the nanofiber membrane with PDMS, the thickness of the nanofiber membrane was obtained by using Image J. Statistical differences between compared groups were determined using single factor ANOVA with a $p$-value less than 0.05 indicating significance.

The mechanical property and structural integrity of the fabricated free-standing nanofiber membrane integrated on the well insert were characterized by a lab-made indentation test machine. Samples fabricated with different electrospinning times of 1, 3, and $5 \mathrm{~min}$, which resulted in different thicknesses of the nanofiber membranes, were prepared. When preparing the samples for indentation test, the concentration of PCL 
solution was set to $7 \mathrm{wt} \%$ to fix the diameter of the nanofibers. The nanofibrous well insert was firmly fixed with a holder in an appropriate position where the centerline of nanofiber membrane was aligned with an indenter rod. The indentation test machine measured the indentation force on a hemispheric indenter imposing a downward movement of the Teflon hemisphere indenter rod $(3 \mathrm{~mm}$ in diameter). The hemispheric indenter, which was connected to a load cell, was moved downward at a constant speed $\left(20 \mu \mathrm{m} \mathrm{s}^{-1}\right)$ by a motorized stage until the nanofiber membrane was ruptured.

\subsection{Cell culture and live/dead assay}

Immortalized mouse bEnd.3 from American Type Culture Collection (Manassas) was cultured in Dulbecco's modified Eagle's medium (Hyclone) with $10 \%$ fetal bovine serum (Hyclone) and 1\% penicillin/streptomycin (Gibco). Cells were maintained in a humidified cell culture incubator at $37{ }^{\circ} \mathrm{C}$ in $5 \% \mathrm{CO}_{2}$ and used before passage number 30 for cell culture experiments. The PCL nanofibrous well insert samples were fabricated with $7 \mathrm{wt} \%$ PCL solution for 5 min electrospinning time. Before seeding the cells on both the PET porous membrane of original Transwell ${ }^{\circledR}$ insert and the PCL nanofiber membrane of nanofibrous well insert, the substrates were sterilized in $70 \%$ ethanol for $2 \mathrm{~h}$, rinsed three times in $1 \times$ phosphate-buffered saline (PBS, Hyclone), and then exposed overnight to UV. Both the membranes were then coated with fibronectin $\left(10 \mu \mathrm{g} \mathrm{mL} \mathrm{mL}^{-1}\right.$, Sigma-Aldrich) for $2 \mathrm{~h}$ in an incubator at $37^{\circ} \mathrm{C}(n=4)$. Cells were seeded on luminal sides of both membranes at a specific density of $1.0 \times 10^{5}$ cells cm$^{-2}$ and cultured for 5 days to reach a confluent monolayer. After culturing for 5 days, cells were rinsed with $1 \times$ PBS and subsequently stained with ethidium homodimer-1 and Calcein AM (LIVE/DEAD Viability kit, Molecular Probes $\left.{ }^{\circledR}\right)$ to compare the cell viability on both membranes. Fluorescent images were acquired using a phase-contrast inverted fluorescence microscope (Nikon TS100F).

\subsection{TEER measurement}

TEER was measured to assess the formation of tight junction and the integrity of endothelial cell monolayer on the membranes using an EVOM2, Epithelial Volt/Ohm Meter and STX3, Chopstick Electrode Set (World Precision Instruments). The TEER value was calculated by subtracting the resistance of the blank from the resistance of a cell-cultured insert every day. Then, the measured TEER values were divided by the area of the membrane $\left(1.12 \mathrm{~cm}^{2}\right)$, and the average TEER value was analyzed for 5 days (total $n=6$ ).

\section{Results and discussion}

\subsection{One-step fabrication process of a nanofibrous well insert}

The one-step process for fabrication of a tunable nanofibrous well insert by adopting the ELES process in this study is schematically shown in Fig. 1a. Owing to the PDMS block that effectively sealed the top opening of the well insert wall, the well

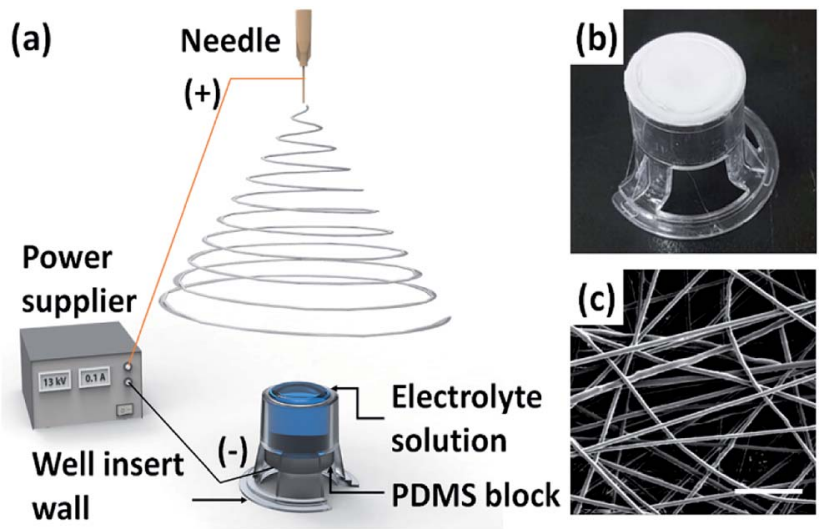

Fig. 1 The one-step fabrication process for a nanofibrous well insert by adopting electrolyte-assisted electrospinning (ELES): (a) a schematic diagram of the experimental set-up, (b) a photograph of the fabricated nanofibrous well insert and (c) SEM image of electrospun nanofiber membrane on the insert. Scale bar represents $5 \mu \mathrm{m}$.

insert wall could securely contain the electrolyte solution, which is to be utilized as a collector in the ELES process. The same volume of the electrolyte solution was poured into the well insert wall placed upside down to maintain the same meniscus shape of the electrolyte solution, preventing variation between the samples. The electrolyte solution played the role of a metal electrode, which is typically used as a grounded collector in electrospinning. Moreover, the electrolyte solution was found to concentrate the electric field to deposit nanofibers selectively on the electrolyte solution and thereby enabled one-step fabrication of nanofibers on the bottom opening of the well insert wall. After electrospinning, the PDMS block was uncapped and the electrolyte solution could be readily removed owing to the fluidic features of the electrolyte solution (Fig. S1, ESI†). The removable characteristic of the electrolyte solution enabled the facile fabrication and spontaneous integration of a freestanding nanofiber membrane directly on the well insert wall, whereas the conventional electrospinning setup inherently needs post-processes, such as peeling-off, transfer, cut, and attachment. The characteristics of fabricated nanofibrous well insert are compared with conventional Transwell inserts, as shown in Table 1. Fig. $1 \mathrm{~b}$ and $\mathrm{c}$ show the fabricated nanofibrous well insert and a representative SEM image of the free-standing nanofiber membrane on it, respectively. The fabrication process provides a facile method of generating the free-standing nanofiber membrane directly on the commercially available Transwell ${ }^{\circledR}$ insert, which possesses easy accessibility and costeffectiveness. By examining the SEM images, the structure of the fabricated nanofiber membrane was found to have an in vivo ECM-like nanofibrous structure, whereas the porous membrane of commercially available Transwell ${ }^{\circ}$ insert possesses randomly distributed micro-sized pores in a flat polymer substrate, which could not directly reflect the in vivo cellular microenvironment. Considering the fact that the free-standing nanofiber membrane in the nanofibrous well insert compartmentalizes the space into two different regions as the porous membrane in the well insert does, the researchers found that 
Table 1 Comparison of PCL nanofibrous well insert with commercially available Transwell@ inserts made of PET and PC provided from Corning Inc. ${ }^{25}$

\begin{tabular}{llll}
\hline Characteristics & Polyester (PET) & Polycarbonate (PC) & Polycaprolactone (PCL) nanofiber \\
\hline Structure & Micro-porous & Micro-porous & Nanofibrous (ECM mimetic) \\
Optical properties & Clear & Translucent & Translucent when wet \\
Membrane thickness $(\mu \mathrm{m})$ & 10 & 10 & $10-80$ (tunable) \\
Available pore sizes $(\mu \mathrm{m})$ & $0.4,1.0,3.0,8.0$ & $0.4,3.0,5.0,8.0$ & $0.4-0.9$ (tunable)
\end{tabular}

the nanofibrous well insert is expected to provide opportunities to develop more in vivo-like cell migration and co-culture model with the ECM-mimicking environment.

\subsection{Characterization of nanofiber membrane}

3.2.1. Tuning of nanofiber membrane properties. Electrospun nanofiber membranes have been known to provide an ECM-mimetic environment. ${ }^{16,17}$ Moreover, modifying the physical properties and the topographical features has been known to significantly influence cell morphology and functions. ${ }^{18-20}$ In this work, three different types of nanofiber membranes were fabricated by the ELES process on the well insert wall while changing the PCL solution concentration from $6 \mathrm{wt} \%$ to $8 \mathrm{wt} \%$, with a fixed electrospinning time of $90 \mathrm{~s}$, as shown in Fig. 2. From the SEM examination on the fabricated nanofiber membranes, the nanofiber diameters of $240 \pm 80,300 \pm 80$, and $510 \pm 190 \mathrm{~nm}$ were fabricated with PCL concentrations of 6,7 , and $8 \mathrm{wt} \%$, respectively, as plotted in Fig. 3a. The result can be explained by an increase in the concentration of polymer solution causing a slower elongation rate and resulting in a thicker nanofiber compared with the case of lower polymer concentration. ${ }^{21}$ Indeed, it was found that a $10 \mathrm{wt} \%$ PCL solution generated microscale electrospun fibers $(1250 \pm 310 \mathrm{~nm})$. The further increase in the concentration of PCL solution to $12 \mathrm{wt} \%$, however, caused the clogging problem at the needle tip and generated instability in electrospinning process, which brought about a significantly high deviation of diameter of electrospun nanofibers $(1700 \pm 710 \mathrm{~nm})$, (Fig. S2, ESI $\dagger$ ).

The SEM examination also revealed that the nanofiber membranes have different pore sizes of $0.4,0.7$, and $0.9 \mu \mathrm{m}$ for the cases of the PCL concentrations of 6,7 , and $8 \mathrm{wt} \%$, respectively, as shown in Fig. $3 \mathrm{~b}$. As a result, the modification of the diameter and the pore sizes of electrospun nanofibers could be achieved by controlling the concentration of the polymer solution. Although there were no statistical differences between data, we could observe the tendency that the pore sizes increased with the PCL solution concentration.

The control over the thickness of electrospun nanofiber membranes could also be accomplished by modulating the
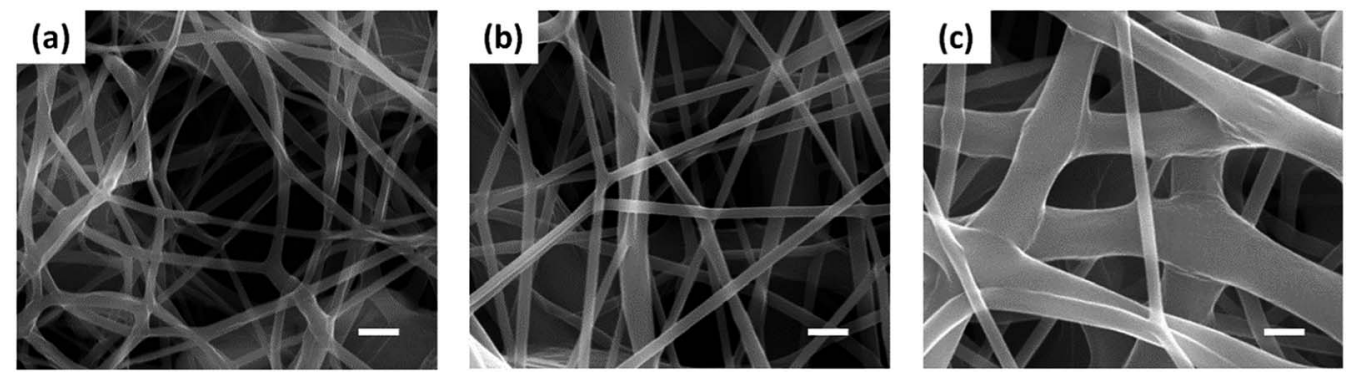

Fig. 2 SEM images of electrospun PCL nanofiber membranes fabricated with PCL aqueous solutions with concentrations of 6 (a), 7 (b), and 8 wt\% (c). Scale bar represents $1 \mu \mathrm{m}$.
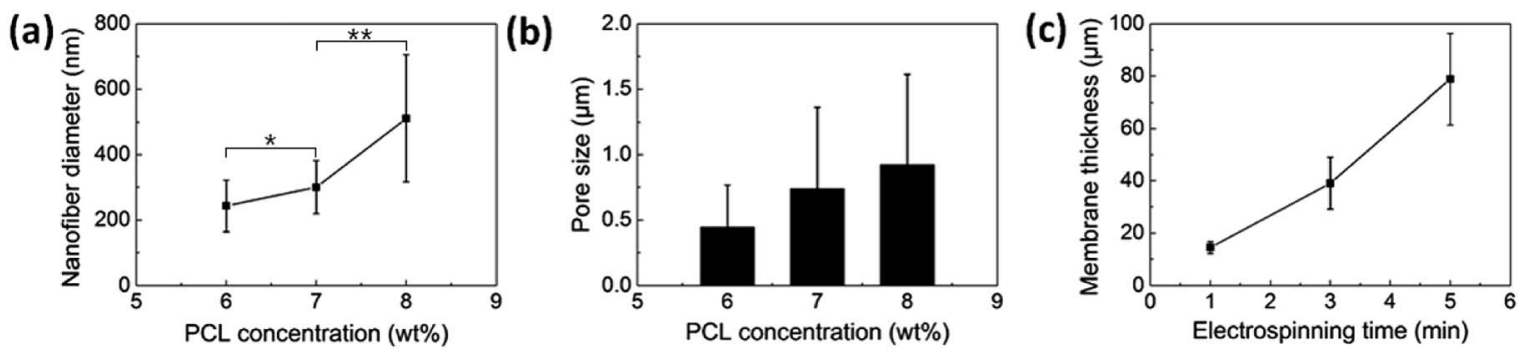

Fig. 3 Modulation of the properties of electrospun nanofiber membrane: nanofiber diameter variation (a) and pore size variation (b) with respect to the PCL concentration. (c) Membrane thickness variation with respect to the electrospinning time. Error bar presents the standard deviations of measured values. 
electrospinning time. As plotted in Fig. 3c, the nanofiber membranes with different thicknesses of $15 \pm 2,39 \pm 10$, and $79 \pm 17 \mu \mathrm{m}$ were fabricated at different electrospinning times of 1,3 , and $5 \mathrm{~min}$, respectively, with the fixed concentration of the PCL solution of $7 \mathrm{wt} \%$. The longer the electrospinning time, the more electrospun nanofibers deposited, which resulted in a thicker nanofiber membrane.

3.2.2. Mechanical property and structural integrity of nanofiber membrane. The mechanical indentation test enabled us to evaluate the fabrication of a structurally stable, freestanding nanofiber membrane and the fabricated membrane firmly attached to the well insert wall using the ELES process. The experimental set-up and the schematic illustration of the indentation test to evaluate the mechanical property of the nanofiber membrane are shown in Fig. $4 \mathrm{a}$ and b, respectively. Fig. 4c shows the curves of the measured force with respect to the displacement of the membrane, which represents the correlation between the electrospinning time and the force required to deform the membrane. Given the increase in the electrospinning time induced to generate the thicker membrane, the force required to deform the membrane was also increased. Similarly, the fracture point of the graph in Fig. 4c was impeded as the electrospinning time increased. During the indentation test, we firm integration of the electrospun nanofiber membrane with the well insert wall.

When we assume that the nanofiber membrane is a freestanding circular thin film with a Poisson's ratio of 0.5 , the deformation of the thin film is known to follow the simplified mechanics model at the large deformation by a hemisphere indenter as shown below: ${ }^{22}$

$$
\frac{\delta}{R}=\left(\frac{a}{R}\right)^{3 / 4}\left(\frac{16}{9 \pi} \frac{R}{E h R}\right)^{1 / 3}
$$

where $R$ is the radius of the indenter, $\delta$ is the displacement, $a$ and $h$ are the radius and thickness of the thin film,
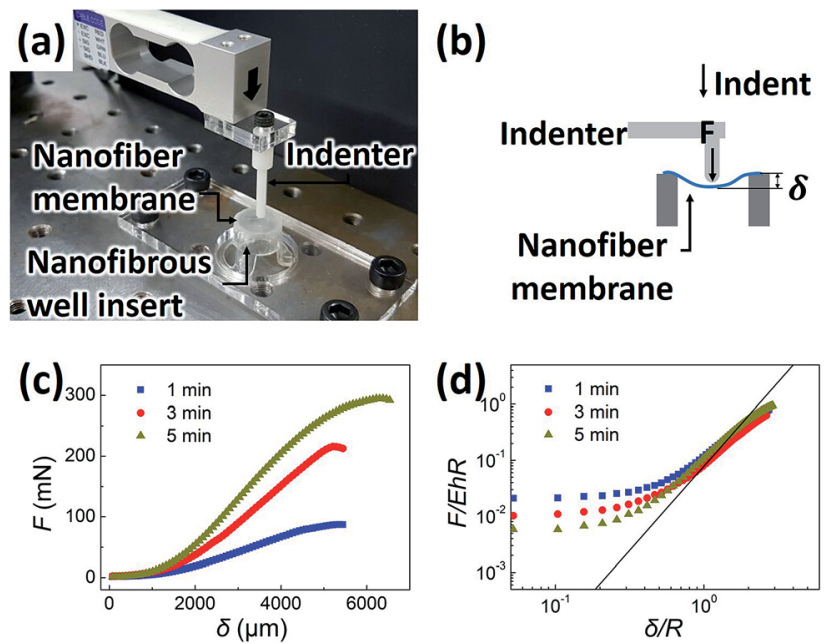

Fig. 4 Mechanical indentation test: (a) a photograph of experimental set-up, (b) the schematic of indentation test, (c) measured indentation force of nanofiber membrane with respect to the displacement, and (d) logarithmic plot of normalized force as a function of the normalized displacement of the nanofiber membrane. respectively, $E$ is a Young's modulus, and $F$ is the applied force. Eqn (1) shows that the force $(F)$ normalized by $E, h$, and $R$ has a cubic relationship with the displacement $(\delta)$ normalized by $R$. In this analysis, we assumed that the nanofiber mat has a Poisson's ratio of exactly 0.5 whose value is for a perfectly incompressible material. Given that the actual Poisson's ratio of the electrospun nanofiber mat would be deviated from the ideal value of 0.5 , this assumption may become a source of error. A cubic relationship between the normalized force and the normalized displacement of the nanofiber membrane on the well insert wall could be demonstrated by drawing a linear regression line in the logarithmic plot, satisfying eqn (1) with the given parameter values, as shown by the black line in Fig. 4d. From the regression line fitted to the experimental data obtained from three different samples, the Young's modulus of the nanofiber membrane was found to be $4 \mathrm{MPa}$. The cubic relationship obtained from the measured force and displacement curves of nanofiber membranes also obviously indicated that the electrospun nanofiber membrane can be regarded as a thin film, not disconnected individual nanofibers.

\subsection{Biological evaluation of nanofibrous well insert}

To validate the biological relevance of the fabricated nanofibrous well inserts as a cell culture platform, we cultured bEnd. 3 cells on the nanofibrous well inserts (12-well dish type, $0.7 \mu \mathrm{m}$ pore size, $300 \mathrm{~nm}$ nanofiber diameter) as well as the commercially available Transwell@ inserts (12-well dish type, $0.4 \mu \mathrm{m}$ pore size, Corning Inc.). The bEnd. 3 cells were cultured on both the inserts for 5 days and stained with live/dead assay at day 5 of culture. The fluorescent image of the cell layer on the nanofibrous well insert showed the comparable viability result
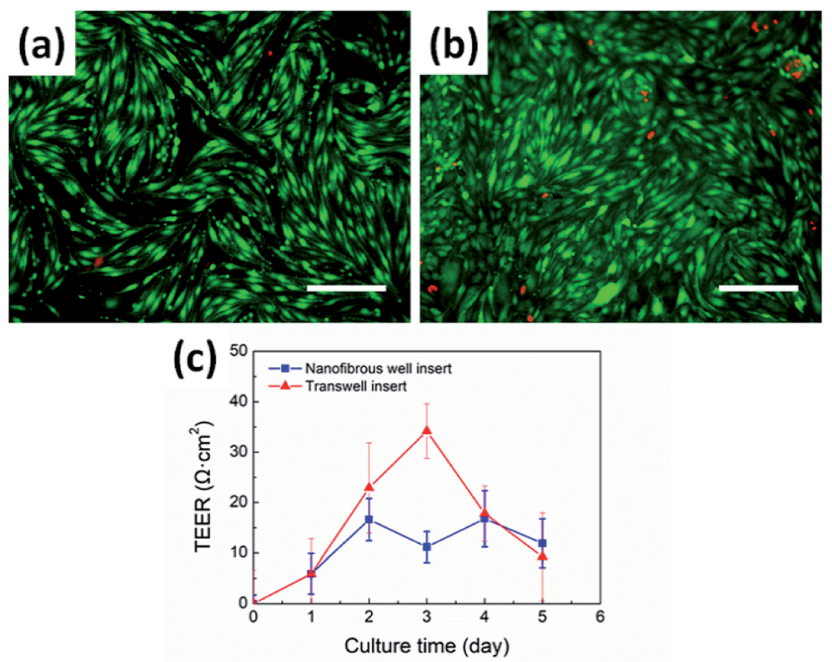

Fig. 5 Evaluation on the biological relevance of nanofibrous well insert compared with commercially available Transwell ${ }^{\circledR}$ insert: (a) live-dead staining of bEnd.3 cell layers cultured on Transwell insert and (b) nanofibrous well insert. (c) Trans-endothelial electrical resistance (TEER) of the bEnd. 3 cell layers cultured on Transwell inserts and nanofibrous well inserts. Live cells were stained green and dead/ damaged cells were strained red at day 5. Scale bar represents $200 \mu \mathrm{m}$ ( $a$ and $b$ ). 
with the well insert, as shown in Fig. 5a and b. The result demonstrated that the bEnd. 3 cells were well-attached to the electrospun PCL nanofiber membrane and proliferated for 5 days. Thus, the nanofibrous well insert could be regarded as a potential cell culture platform, which provides a more ECMmimetic cell microenvironment compared with the conventional 2D flat culture surfaces. ${ }^{16,17}$

TEER value was measured to evaluate the formation of the tight junction among the endothelial cells and the integrity of the cell layer, as shown in Fig. 5c. Given that an increase in TEER value indirectly represents the integrity of the cell layer, ${ }^{23,24}$ the continuation of escalated TEER value of the nanofibrous well insert after cell culture could be regarded as the formation of the tight junction. In addition, considering the TEER value of the nanofibrous well inserts, with the well inserts having no statistical difference $(P<0.05)$ at day 5 of culture, we conclude that the nanofibrous well inserts performed a comparable role with the commercially available well inserts to form a tight junction among the endothelial cells. As seen in Fig. 5c, the TEER value of well insert peaked at day 3 of culture. Given that the integrity of bEnd.3 monolayer decreases after forming a confluent layer, the peak implies that the cells reached the confluent state after 2 days to 3 days of cell seeding on the well insert. The non-uniform nanofibrous membrane surface might affect the reduced TEER value compared with the conventional flat porous membrane at day 3 of culture. Such interesting behavior should further be studied in future work. With the demonstrated biological capability, the present nanofibrous well inserts can be applied to various fields including immune response, wound healing, and nutrient and protein transports, providing a more ECM-mimetic environment compared with the conventional $2 \mathrm{D}$ cell culture platforms.

\section{Conclusions}

Electrospinning provides the simplest method to fabricate nanofibrous structures that in turn provide ECM-mimicking cell microenvironment. The application of electrospun nanofibers on in vitro cell culture platforms would create a deeper understanding of various pathologies. The present work suggested a one-step fabrication process of a nanofibrous well insert wherein the electrospun nanofiber membrane is integrated into the well insert wall by adopting the ELES process. The ELES process not only eliminated the complexity in producing the nanofibrous well insert by directly fabricating a nanofiber membrane on the insert wall but also spontaneously integrated the membrane on the insert wall in a one-step fabrication process. It also provided a versatile method to modify the properties of the nanofiber membranes, such as the diameter of the nanofibers and porosity and thickness of the nanofiber membrane. The nanofiber membrane was found to be utilized as a potential cell culture platform that provides the ECM-mimicking environment. Therefore, we believe that the present one-step fabrication process of nanofibrous well inserts will facilitate the utilization of the nanofiber membrane in various biomedical applications.

\section{Conflicts of interest}

There are no conflicts of interest to declare.

\section{Acknowledgements}

This research was supported by the National Research Foundation of Korea (NRF) grant funded by the Korea Government (MSIP) (No. 2017R1A2A1A05001090 and No. 2011-0030075).

\section{Notes and references}

1 A. E. Karnoub, A. B. Dash, A. P. Vo, A. Sullivan, M. W. Brooks, G. W. Bell, A. L. Richardson, K. Polyak, R. Tubo and R. A. Weinberg, Nature, 2007, 449, 557-563.

2 S. F. Tavazoie, C. Alarcón, T. Oskarsson, D. Padua, Q. Wang, P. D. Bos, W. L. Gerald and J. Massagué, Nature, 2008, 451, 147-152.

3 R. Förster, A. Schubel, D. Breitfeld, E. Kremmer, I. RennerMüller, E. Wolf and M. Lipp, Cell, 1999, 99, 23-33.

4 N. E. Hastings, M. B. Simmers, O. G. McDonald, B. R. Wamhoff and B. R. Blackman, Am. J. Physiol.: Cell Physiol., 2007, 293, C1824-C1833.

5 K. S. Moulton, K. Vakili, D. Zurakowski, M. Soliman, C. Butterfield, E. Sylvin, K.-M. Lo, S. Gillies, K. Javaherian and J. Folkman, Proc. Natl. Acad. Sci. U. S. A., 2003, 100, 4736-4741.

6 W.-S. Kim, B.-S. Park, J.-H. Sung, J.-M. Yang, S.-B. Park, S.-J. Kwak and J.-S. Park, J. Dermatol. Sci., 2007, 48, 15-24.

7 L. Chen, E. E. Tredget, P. Y. Wu and Y. Wu, PLoS One, 2008, 3, e1886.

8 M. Maresca, R. Mahfoud, N. Garmy and J. Fantini, J. Nutr., 2002, 132, 2723-2731.

9 P. H. Warnke, M. Alamein, S. Skabo, S. Stephens, R. Bourke, P. Heiner and Q. Liu, Acta Biomater., 2013, 9, 9414-9422.

10 L. L. Bischel, P. N. Coneski, J. G. Lundin, P. K. Wu, C. B. Giller, J. Wynne, B. R. Ringeisen and R. K. Pirlo, J. Biomed. Mater. Res., Part A, 2016, 104, 901-909.

11 S. C. Slater, V. Beachley, T. Hayes, D. Zhang, G. I. Welsh, M. A. Saleem, P. W. Mathieson, X. Wen, B. Su and S. C. Satchell, PLoS One, 2011, 6, e20802.

12 S. M. Park and D. S. Kim, Adv. Mater., 2015, 27, 1682-1687.

13 C. J. Luo, E. Stride and M. Edirisinghe, Macromolecules, 2012, 45, 4669-4680.

14 X. M. Mo, C. Y. Xu, M. Kotaki and S. Ramakrishna, Biomaterials, 2004, 25, 1883-1890.

15 M. S. Kim, J. Son, H. Lee, H. Hwang, C. H. Choi and G. Kim, Curr. Appl. Phys., 2014, 14, 1-7.

16 Z. Ma, M. Kotaki, R. Inai and S. Ramakrishna, Tissue Eng., 2005, 11, 101-109.

17 Q. P. Pham, U. Sharma and A. G. Mikos, Tissue Eng., 2006, 12, 1197-1211.

18 J. S. Choi, S. J. Lee, G. J. Christ, A. Atala and J. J. Yoo, Biomaterials, 2008, 29, 2899-2906.

19 C. Xu, R. Inai, M. Kotaki and S. Ramakrishna, Biomaterials, 2004, 25, 877-886. 
20 B.-M. Min, G. Lee, S. H. Kim, Y. S. Nam, T. S. Lee and W. H. Park, Biomaterials, 2004, 25, 1289-1297.

21 C. Thompson, G. G. Chase, A. Yarin and D. Reneker, Polymer, 2007, 48, 6913-6922.

22 M. R. Begley, H. Bart-Smith, O. N. Scott, M. H. Jones and M. L. Reed, J. Mech. Phys. Solids, 2005, 53, 2557-2578.
23 H. E. de Vries, M. C. Blom-Roosemalen, M. van Oosten, A. G. de Boer, T. J. van Berkel, D. D. Breimer and J. Kuiper, J. Neuroimmunol., 1996, 64, 37-43.

24 N. J. Abbott, L. Rönnbäck and E. Hansson, Nat. Rev. Neurosci., 2006, 7, 41-53.

25 Transwell Permeable Supports Selection and Use Guide, http:// csmedia2.corning.com/LifeSciences/Media/pdf/transwell_ guide.pdf, accessed May 2017. 